\title{
Ongoing outbreak of invasive listeriosis, Germany, 2012 to 2015
}

W Ruppitsch ${ }^{12}$, R Prager ${ }^{23}$, S Halbedel ${ }^{3}$, P Hyden ${ }^{1}$, A Pietzka ${ }^{1}$, S Huhulescu ${ }^{1}$, D Lohr 45 , K Schönberger ${ }^{6}$, E Aichinger ${ }^{4}$, A

Hauri $^{7}$, K Stark $^{8}$, S Vygen $^{8}$, E Tietze $^{3}$, F Allerberger $^{1}$, H Wilking $^{8}$

1. German-Austrian Binational Consiliary Laboratory for Listeria, Austrian Agency for Health and Food Safety (AGES), Vienna, Austria

2. These authors contributed equally

3. Division Enteropathogenic Bacteria and Legionella, Robert Koch Institute (RKI), Wernigerode, Germany

4. Baden-Wuerttemberg State Health Office, Stuttgart, Germany

5. European Programme for Intervention Epidemiology Training (EPIET), European Centre for Disease Prevention and Control (ECDC), Stockholm, Sweden

6. Bavarian Health and Food Safety Authority (LGL), Oberschleißheim, Germany

7. Hesse State Health Office, Dillenburg, Germany

8. Division for Gastrointestinal Infections, Zoonoses and Tropical Infections, Robert Koch Institute (RKI), Berlin, Germany

Correspondence: Hendrik Wilking (wilkingh@rki.de)

Citation style for this article:

Ruppitsch W, Prager R, Halbedel S, Hyden P, Pietzka A, Huhulescu S, Lohr D, Schönberger K, Aichinger E, Hauri A, Stark K, Vygen S, Tietze E, Allerberger F, Wilking H. Ongoing outbreak of invasive listeriosis, Germany, 2012 to 2015. Euro Surveill. 2015;20(50):pii=30094. DOI: http://dx.doi.org/10.2807/1560-7917.

ES.2015.20.50.30094

Listeriosis patient isolates in Germany have shown a new identical pulsed-field gel electrophoresis (PFGE) pattern since $2012(\mathrm{n}=66)$. Almost all isolates (Listeria monocytogenes serotype $1 / 2 a$ ) belonged to cases living in southern Germany, indicating an outbreak with a so far unknown source. Case numbers in 2015 are high $(n=28)$. No outbreak cases outside Germany have been reported. Next generation sequencing revealed the unique cluster type CT1248 and confirmed the outbreak. Investigations into the source are ongoing.

Since November 2012, a previously not observed pulsed-field gel electrophoresis (PFGE) pattern in human isolates of invasive L. monocytogenes serotype $1 / 2$ a has been detected in Germany with increasing frequency. Altogether 66 outbreak cases have been recorded, with 28 cases in 2015 . Four cases were pregnancy-associated and six cases died in the course of the disease. Here we provide details of the ongoing outbreak.

\section{Outbreak description}

Since 2009, all German Listeria isolates submitted to the National Reference Centre (NRC) for Salmonella and other bacterial enterics at the Robert-Koch Institute (RKI) or to the Austrian-German binational reference laboratory $(\mathrm{KL})$ for Listeria at the Austrian Agency for Health and Food Safety (AGES), have been tested with PFGE for clonal relationship. Submission of isolates is encouraged by public health authorities but is voluntary without legal obligation. Between November 2012 and November 2015, altogether 793 isolates from notified listeriosis cases were typed, which accounted for
$45 \%$ of all cases in that period $(n=1,765)$. In southern Germany, this proportion was higher (ca 60\%) and since 2012 , human isolates of $L$. monocytogenes serotype $1 / 2 a$ with the NRC internal nomenclature of the Ascl/Apal pattern 13a/54 have been observed.

By 30 November 2015, the typing centres had received a total of 69 isolates with the 13a/54 PFGE pattern. Multilocus sequence typing (MLST) revealed sequence type 8 (www.pasteur.fr/mlst). After exclusion of three isolates (see below), next generation sequencing (NGS) was applied to 38 of 66 isolates using a published core genome MLST (cgMLST) [1]. All 38 patient isolates could be allocated to one cluster type (CT1248) (Figure 1).

We used the following case definition in our investigation: Possible outbreak cases were patients with the clinical picture of acute invasive listeriosis with onset since November 2012 with isolation of Listeria from normally sterile body fluids and detection of the characteristic PFGE pattern 13a/54. Confirmed cases were patients meeting the above criteria with isolates assigned to cluster CT1248 in NGS.

According to the Protection Against Infection Act of 2001, laboratory confirmation of Listeria from a normally sterile site is notifiable to local health departments which transmit information to RKI. Of the 69 isolates with the $13 a / 54$ PFGE pattern, 66 could be assigned to surveillance cases reported in the mandatory notification system; of those, 38 were confirmed by NGS. Figure 2 illustrates the outbreak cases by month. There was a first peak in the second half of 


\section{FIGURE 1}

Minimum Spanning Tree based on NGS allelic profiles of Listeria monocytogenes isolates, Germany, 2012-15 (n=160)

A.

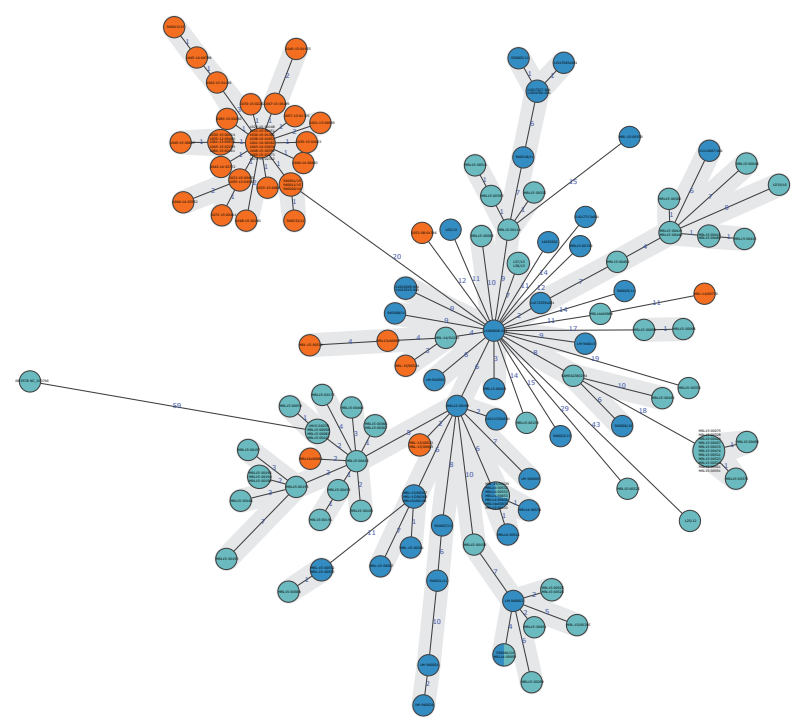

PFGE $13 a / 54$

PFGE One band difference in Apal

PFGE other similar
B.

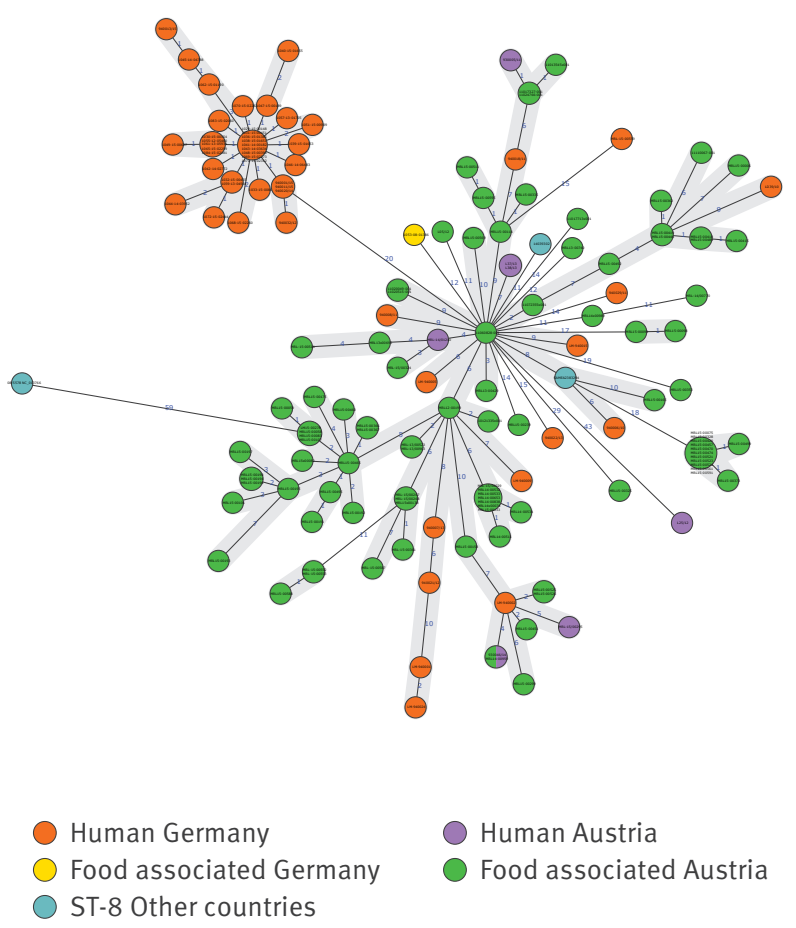

PFGE: pulsed-field gel electrophoresis.

After the evaluation scheme in [1]. Panel A: Sequence-based clonal relationship stratified for pulsed-field pattern designation to PFGE type 13a/54, a similar PFGE type with one band difference in Apal and other similar PFGE types). Panel B: Stratified for the origin (food-borne case) of isolates. Each circle represents an allelic profile based on sequence analysis of 1,701 target genes. The numbers on the connecting lines illustrate the numbers of target genes with differing alleles. The different groups of strains are distinguished by the colours of the circles. Closely related genotypes ( $>10$ allele difference) are shaded in grey and designated cluster type.

\section{FIGURE 2}

Temporal distribution of listeriosis outbreak cases (28 possible and 38 confirmed) with PFGE pattern 13a/54 and available notification date, Germany, 2012-15 $(\mathrm{n}=66)$

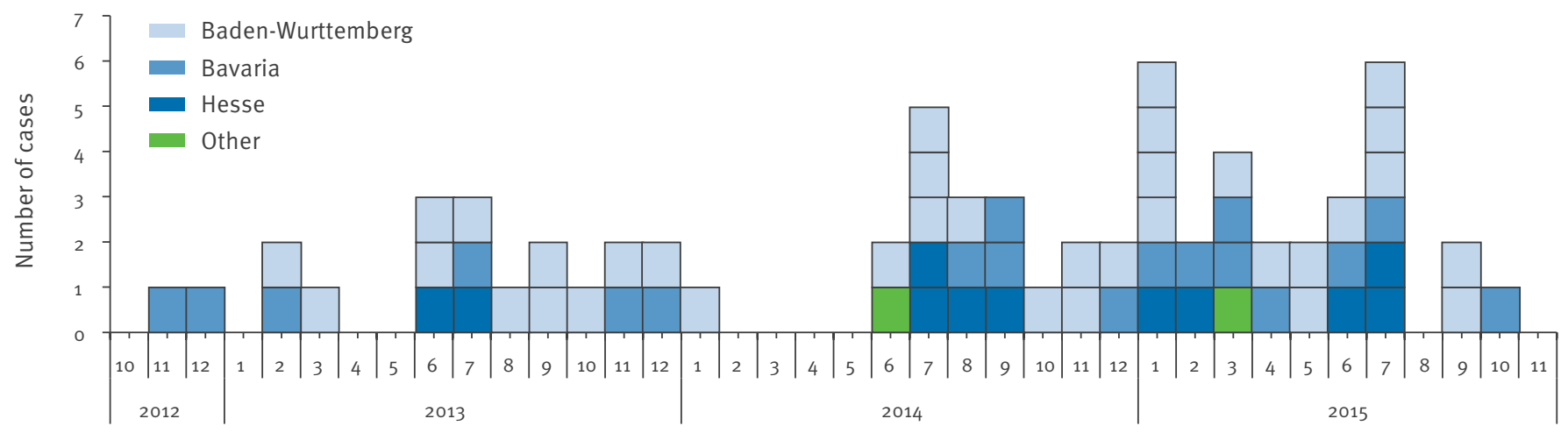

Year and month of notification

PFGE: pulsed-field gel electrophoresis. 


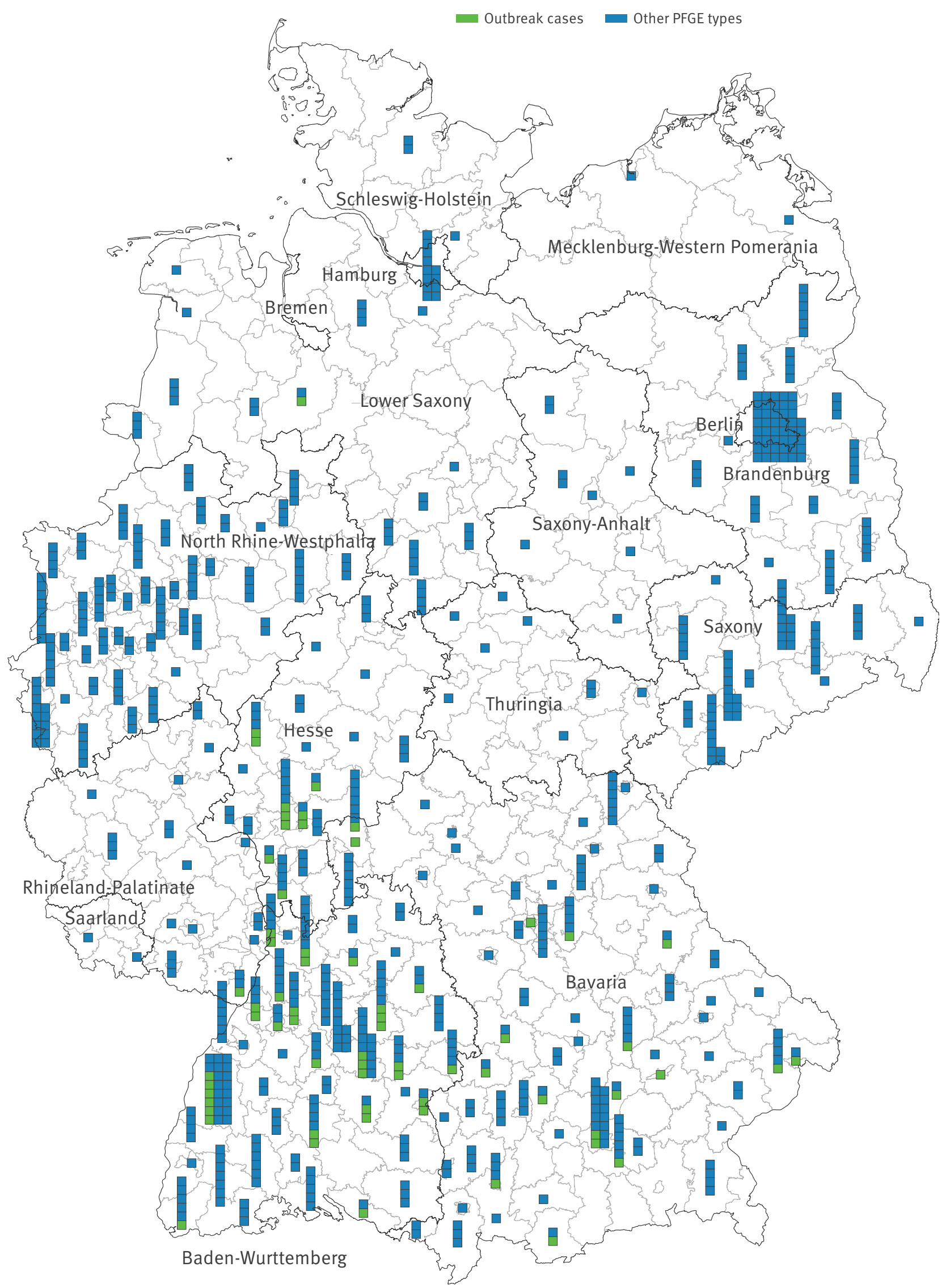


2013, but most cases have occurred since June 2014 (compared with a total of 609 invasive listeriosis cases in Germany in 2014). In 2015, this has so far been the most frequently occurring PFGE pattern among all Listeria isolates in molecular surveillance.

The geographical distribution was largely confined to the states of Baden-Wuerttemberg, Bavaria and Hesse, although PFGE typing is also frequently applied for isolates from the north of Germany (Figure 3). Only one case each was reported from Rhineland-Palatinate and Lower Saxony.

Four of the 66 cases were pregnancy-associated. Among 62 not pregnancy-associated outbreak patients 32 were men. The outbreak affected 38 senior citizens ( $\geq 70$ years), 23 younger adults (18-69 years) and one two-year-old child. They did not differ from other listeriosis surveillance cases not related to the outbreak $(n=1,699)$ with respect to age $(p=0.628)$ and sex $(p=0.433)$. Of the 62 cases, 44 suffered from fever $\geq 38.5^{\circ} \mathrm{C}, 16$ had meningitis, 16 had septicaemia and for 15, other listeriosis-related symptoms were reported. Six (not pregnancy-associated) cases died; three of the deaths were confirmed to be due to listeriosis as the major cause.

This outbreak was communicated via the European Epidemic Intelligence Information System (EPIS) platform on 17 July 2015 and updated on 5 November 2015. None of the other participating countries reported cases with the outbreak PFGE pattern or NGS cluster types.

\section{Investigation into the source of infection}

Initial screening of food-related Listeria isolates in the strain collection of RKI and AGES found a total of six isolates (five from Austria and one from Germany) which had indistinguishable PFGE patterns but belonged to different NGS cluster types (Figure 1). Food consumption histories have been collected from a subset of cases via exploratory interviews by the health authorities since 2013. Furthermore, information on food consumption habits are recorded via collection of patients' grocery receipts [2]. Many patients can have difficulties recalling food consumption because of their age and their disease. Photo documentation of food items regularly purchased by some patients is used for visual support during interviews with other patients. Epidemiological studies were conducted in cooperation with regional and local health departments, considering incubation periods published by Goulet et al. [3].

Regarding the source of the causative food vehicle, the results showed a heterogeneous picture. Until now we have not observed cases with an epidemiological link to an institution (e.g. hospital infection). Preliminary results largely exclude fish and cheese products as a possible source but this has to be complemented by systematic screening of Listeria isolates collected from food. Based on sequencing results, a PCR protocol aiming to detect CT1248 was developed for screening of isolates and published on the KL website [4].

\section{Background}

L. monocytogenes, the causative agent of listeriosis is mostly caused by the consumption of contaminated food. The majority of infections are mild if they occur in younger, immunocompetent individuals except pregnant women. Infection during pregnancy can lead to miscarriage, stillbirth and serious health problems for the newborn. Invasive listeriosis can cause severe septicaemia, meningoencephalitis and a wide variety of focal infections. It is usually limited to the elderly and those with compromised immune systems or severe underlying medical conditions. Because of the severity of certain clinical manifestations (infections of the central nervous system, septicaemia and abortion), the high case-fatality rate of up to $30 \%$ and the long incubation time, human listeriosis is of major public health concern. A recent nationwide case-control study in Germany among sporadic disease cases detected cold cooked sausages, packaged cheese and pre-sliced cheese as risk foods [5]. Medical conditions associated with listeriosis are immunosuppressive therapy, immunocompromising disease and gastric acid suppression [5].

\section{Public health assessment}

When considering confirmed as well as possible cases, this is the largest outbreak of listeriosis described in Germany to date [6]. Considering underascertainment, under-reporting and the considerable proportion of isolates that are not typed, the size of the visible outbreak of invasive listeriosis is certainly underestimated. Furthermore, mild and non-invasive gastrointestinal cases, which can make up a significant proportion of disease cases, are not under surveillance in Germany. Until now, the cluster type CT1248 is confined to this outbreak and investigation via EPIS did not generate feedback on isolates with a related sequence in participating countries. Listeriosis cases have become more frequent over the past years in Germany [7] and elsewhere in Europe [8]. Investigations of listeriosis outbreaks are difficult due to the multitude of possible food vehicles including a broad range of ready-to-eat foods.

PFGE is suitable for screening but cannot confirm outbreak isolates, whereas NGS appears highly discriminatory and superior for the allocation of cases to the outbreak. The geographical limitation to southern Germany and the size of the outbreak area with a population of 27 million inhabitants suggest Listeriacontaminated food in a supra-regional supermarket grocery chain as the vehicle of infection. Although the number of new cases has decreased since August 2015, new outbreak cases are still being reported. We must therefore assume that the source of infection is still active and further cases are possible. Further epidemiological studies, laboratory investigations and 
trace-back of food items are needed and ongoing to narrow down the source of infection.

Diagnostic laboratories are requested to send any Listeria isolates to one of the typing centres. The use of NGS is desirable as routine for all Listeria isolates collected for typing.

\section{Acknowledgements}

The work was funded by the authors' employers. We gratefully acknowledge the contribution of all local and state health departments. We thank the Federal Institute for Risk Assessment (BfR) and the Federal Office of Consumer Protection and Food Safety (BVL) as partners in the ongoing investigation into the outbreak source, and Karina Preußel and Niels Kleinkauf for their help.

\section{Conflict of interest}

None declared.

\section{Authors' contributions}

Wrote the manuscript: HW; performed epidemiological analysis: HW, SV; supervised outbreak: FA, ET, KS; regional surveillance: EA, DL, AH, KS; performed laboratory investigation: RP, AP; ET, PH, MB; performed phylogenetic analyses: WR, SH; all authors revised the manuscript.

\section{References}

1. Ruppitsch W, Pietzka A, Prior K, Bletz S, Fernandez HL, Allerberger F, et al. Defining and Evaluating a Core Genome Multilocus Sequence Typing Scheme for Whole-Genome Sequence-Based Typing of Listeria monocytogenes. I Clin Microbiol. 2015;53(9):2869-76. DOI: 10.1128/JCM.01193-15 PMID: 26135865

2. Fretz R, Sagel U, Ruppitsch W, Pietzka A, Stoger A,

Huhulescu S, et al. Listeriosis outbreak caused by acid curd cheese Quargel, Austria and Germany 2009. Euro Surveill. 2010;15(5):pii=19477.PMID: 20144447

3. Goulet V, King LA, Vaillant V, de Valk H. What is the incubation period for listeriosis?BMC Infect Dis. 2013;13(1):11. DOI: 10.1186/1471-2334-13-11 PMID: 23305174

4. Austrian Agency for Health and Food Safety (AGES). TaqMan Assay zum Screenen von Listeria monocytogenes Isolaten auf CT1248. Vienna: AGES; 2015.

5. Preußel K, Milde-Busch A, Schmich P, Wetzstein M, Stark K, Werber D. Risk Factors for Sporadic Non-Pregnancy Associated Listeriosis in Germany-Immunocompromised Patients and Frequently Consumed Ready-To-Eat Products.PLoS ONE. 2015;10(11):e0142986. DOI: 10.1371/journal.pone.0142986 PMID: 26599484

6. Koch J, Dworak R, Prager R, Becker B, Brockmann S, Wicke A, et al. Large listeriosis outbreak linked to cheese made from pasteurized milk, Germany, 2006-2007. Foodborne Pathog Dis. 2010;7(12):1581-4. DOI: 10.1089/fpd.2010.0631 PMID: 20807110

7. Robert-Koch Institute (RKI). Infektionsepidemiologisches Jahrbuch meldepflichtiger Krankheiten für 2014. Berlin: RKI; 2015.

8. European Centre for Disease Prevention and Control (ECDC). European Food Safety Authority (EFSA), The European Union summary report on trends and sources of zoonoses, zoonotic agents and food-borne outbreaks in 2013. Parma, Italy and Stockholm, Sweden; 2015. Available from: http://www.efsa. europa.eu/en/efsajournal/pub/3991 